\title{
Dynamic heterodyne near field scattering
}

\author{
D. Magatti, ${ }^{1}$ M. D. Alaimo, ${ }^{2}$ M. A. C. Potenza, ${ }^{2}$ and F. Ferri $\left.{ }^{1}, a\right)$ \\ ${ }^{1}$ Dipartimento di Fisica e Matematica, Università dell'Insubria, Via Valleggio 11, Como I-22100, Italy \\ ${ }^{2}$ Dipartimento di Fisica, Università di Milano, Via Celoria 16, Milano I-20133, Italy
}

(Received 7 March 2008; accepted 8 May 2008; published online 16 June 2008)

\begin{abstract}
The technique heterodyne near field scattering (HNFS), originally developed for low-angle static light scattering, has also been implemented for carrying out dynamic light scattering. While the classical dynamic light scattering method measures the intensity-intensity correlation function, dynamic HNFS gives directly the field-field correlation function, without any assumption on the statistical properties of the sample, as the ones required by the Siegert relation. The technique has been tested with calibrated Brownian particles and its performances compared to those of the classical dynamic light scattering method. (C) 2008 American Institute of Physics.
\end{abstract}

[DOI: $10.1063 / 1.2937841]$

Low-angle light scattering (LALS) is a well established technique for studying mesoscopic disordered systems that present inhomogeneities on length scales comparable to the wavelength of light or larger. Examples of applications of LALS are countless, going from the field of colloidal aggregation to polymer blends, gel formation, and, in general, the chemical physics of complex fluids and critical phenomena. In the past, LALS has been mainly used for carrying out static light scattering, and only recently, with the advent of high resolution two-dimensional (2D) charge coupled device (CCD) sensors, the technique has been implemented to carry out also dynamic light scattering (DLS). ${ }^{1,2}$ LALS is based on the measurement of light scattered in the far field of the sample and exploits the large statistics provided by the huge number of pixels available on modern CCDs. However, there are several drawbacks that limit the technique, the main ones being the removal of the transmitted beam that requires tight constraints on the alignment and the presence of unavoidable forward scattered stray light, which imposes blank measurements and makes the system intrinsically nonergodic. ${ }^{2}$

To overcome these limitations, we adopted a method based on the heterodyne near field scattering (HNFS), ${ }^{3,4}$ a technique that is an extremely valid alternative to classical static LALS. ${ }^{5}$ HNFS works by letting the transmitted beam interfere with scattered light and by detecting the resulting intensity distribution in the near field of the sample. Then, by computing the time-averaged spatial power spectrum of the intensity fluctuations, one recovers directly the static scattered intensity distribution $I(q)$ as a function of the scattering wavevector $q$. HNFS works for $q$ values much larger than a crossover $q^{*}$ that depends on various parameters related to the optical setup (see Fig. 1): the sample thickness $\ell$, the size $L$ of the effective sensor, the distance $z$ between the sample and sensor, and the vacuum wavevector $k$ of the incident beam. For an infinitely large sensor $(L \rightarrow \infty), q^{*}$ is set only by the sample thickness and given ${ }^{6}$ by $q_{\ell}^{*}=\sqrt{\pi k / \ell}$, while for an infinitesimally thin sample $(\ell=0), q^{*}$ is set by the sensor size and given ${ }^{3}$ by $q_{L}^{*}=k L /(2 z)$. Thus, HNFS requires $q \gg q^{*}$ $=\min \left\{q_{\ell}^{*}, q_{L}^{*}\right\}$. Conversely, when $q \ll q^{*}$, the measured power spectrum exhibits deep oscillations (known as the Talbot effect ${ }^{7}$ ) that modulate $I(q)$ and may obscure its behavior ver-

a) Author to whom correspondence should be addressed. Electronic mail: fabio.ferri@uninsubria.it. sus $q$. In this regime, the technique is known as quantitative shadowgraph (QS) and was proposed about a decade ago ${ }^{8,9}$ to analyze quantitatively the shadowgraph images used for studying refraction index fluctuations in transparent fluids. Note that HNFS and QS work exactly in the same manner but in two different $q$ regimes, which are referred, in the context of acousto-optics, as the Bragg and Raman-Nath regimes, respectively. ${ }^{6}$ For completeness, we add that a third method, based onto a Schlieren layout, ${ }^{10}$ has been recently suggested. Such a method works in the QS regime with no oscillations but with non-negligible drawbacks. For a comparison between all these methods, see Ref. 11 .

In this work, we have implemented the HNFS technique showing that, as originally suggested in Ref. 4 and recently carried out in the QS regime, ${ }^{11-13}$ the technique can be fruitfully used for carrying out simultaneous static and dynamic low-angle light scattering. Different from traditional DLS that measures the intensity-intensity correlation function $G_{I}(q, \tau)$ and recovers the field-field correlation function $G_{E}(q, \tau)$ via the Siegert relation, ${ }^{14}$ dynamic HNFS allows for a direct measurement of $G_{E}(q, \tau)$. This is of interest for systems that do not exhibit Gaussian statistics, as it happens at a low angle because of unavoidable stray light. ${ }^{2}$

The HNFS technique requires a simple optical setup (see Fig. 1), in which a large collimated laser beam of diameter $D$ is sent onto a cell containing the sample. By using a CCD sensor and a lens (maximum acceptance angle $2 \theta_{\max }$ ), we record the intensity distribution of the light falling onto a plane at a close distance $z$ from the sample. The near field condition is met when $D \gg D^{*}, D^{*}$ being the size of the region from which the scattered light is collected $\left(D^{*}\right.$ $\left.\sim 2 z \theta_{\max }\right)$. On the observation plane, the interference between the scattered $e_{s}$ and the transmitted field $e_{0}$ gives rise to an intensity distribution that, if $e_{s} \ll e_{0}$, is given by

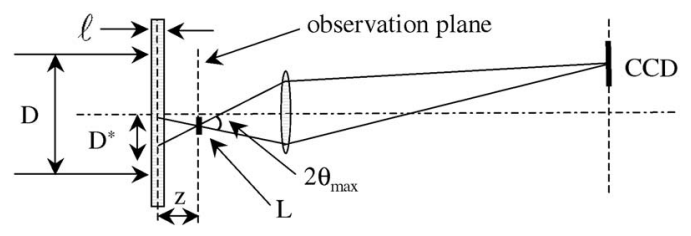

FIG. 1. Sketch of the HNFS setup. 


$$
f(\mathbf{r}, t)=i_{0}(\mathbf{r})+2 \operatorname{Re}\left\{e_{0}^{*}(\mathbf{r}) e_{S}(\mathbf{r}, t)\right\},
$$

where $i_{0}(\mathbf{r})=\left|e_{0}(\mathbf{r})\right|^{2}$ is the static transmitted intensity, while the last term is a zero-average fluctuating term which is called heterodyne signal. If the sample is made of particles, the signal is the sum of many independent patterns originated from the interference between $e_{0}$ and the fields scattered by each particle, whose positions are random. If the particles move because of diffusion, the heterodyne signal varies accordingly and, from its time fluctuations, one can recover the diffusion coefficient of the particles.

The optical setup is sketched in Fig. 1. A cw He-Ne laser $(\lambda=632.8 \mathrm{~nm})$ was spatially filtered, collimated to a diameter $D \sim 10 \mathrm{~mm}$ (at $1 / e^{2}$ of the intensity), and sent onto the sample contained in an $\ell=2 \mathrm{~mm}$ optical path cell. The collecting lens was a $20 \times$ Spindler\&Hoyer microscope objective with a numerical aperture of 0.50 , corresponding to a maximum wavevector $q_{\max } \sim 5.1 \mu \mathrm{m}^{-1}$. The distance between the observation plane and the cell was $z=2 \mathrm{~mm}$. The data were acquired with a 12 bit CCD camera (Vosskuhler CCD-1300, pixel size of $6.67 \mu \mathrm{m}$ ), the exposure time was $\sim 1 \mathrm{~ms}$, and images of $1024 \times 1024$ pixels were used in the Fourier analysis. The side of the effective sensor was $L$ $=341.5 \mu \mathrm{m}$ and the minimum wavevector $q_{\min }=2 \pi / L$ $\sim 1.84 \times 10^{-2} \mu \mathrm{m}^{-1}$. Thus the wavevector $q^{*}$ at which the HNFS regimes starts $\left(q>q^{*}\right)$ is $q^{*}=q_{\ell}^{*} \sim 0.13 \mu \mathrm{m}^{-1}$. The samples were monodisperse latex spheres (Duke Scientific, $\sigma /\langle\mathrm{d}\rangle \sim 1 \%)$ diluted in density matched deuterated water to avoid sedimentation.

The data analysis was carried out by adopting the double frame analysis suggested in Ref. 4, in which a set of $N$ frames sampled every $\Delta t=0.1 \mathrm{~s}$, is first acquired and then processed. By indicating with $f(\mathbf{r}, t)$ and $f(\mathbf{r}, t+\tau)$ two frames taken at a time distance $\tau(\tau=k \Delta t, k$ being an integer), we can define a differential heterodyne signal as

$$
\begin{aligned}
\delta f(\mathbf{r}, t, \tau) & =f(\mathbf{r}, t+\tau)-f(\mathbf{r}, t) \\
& =2 \operatorname{Re}\left\{e_{0}^{*}(\mathbf{r}) e_{s}(\mathbf{r}, t+\tau)-e_{0}^{*}(\mathbf{r}) e_{s}(\mathbf{r}, t)\right\},
\end{aligned}
$$

which is clearly independent of $i_{0}(\mathbf{r})$. If we indicate with $\delta F(\mathbf{q}, t, \tau)$, the spatial Fourier transform of $\delta f(\mathbf{r}, t, \tau)$, the power spectrum associated with this signal is

$$
\begin{aligned}
|\delta F(\mathbf{q}, t, \tau)|^{2} & \\
\sim & |E(\mathbf{q}, t)|^{2}+|E(-\mathbf{q}, t)|^{2}+|E(\mathbf{q}, t+\tau)|^{2}+|E(-\mathbf{q}, t+\tau)|^{2} \\
& -2 \operatorname{Re}\left\{E(\mathbf{q}, t) E^{*}(\mathbf{q}, t+\tau)+E(-\mathbf{q}, t) E^{*}(-\mathbf{q}, t+\tau)\right\} \\
& +2 \operatorname{Re}\{E(\mathbf{q}, t) E(-\mathbf{q}, t)+E(\mathbf{q}, t+\tau) E(-\mathbf{q}, t+\tau)\} \\
& -2 \operatorname{Re}\{E(-\mathbf{q}, t) E(\mathbf{q}, t+\tau)+E(\mathbf{q}, t) E(-\mathbf{q}, t+\tau)\},
\end{aligned}
$$

in which $E(\mathbf{q}, t)$ is the Fourier transform of $e_{S}(\mathbf{r}, t)$ and we have neglected any dependence of $e_{0}$ on $\mathbf{r}$. Note that the terms appearing in lines 1 and 2 involve the products of the fields scattered with the same wavevector (either $+\mathbf{q}$ or $-\mathbf{q}$ ), while in lines 3 and 4 appear only those with opposite vectors $(+\mathbf{q}$ and $-\mathbf{q})$. The latter ones are the shadowgraph terms,${ }^{8,9}$ responsible for the deep oscillations observed in the QS regime. Conversely in the HNFS regime $\left(q \gg q^{*}\right)$, the phase difference between the $+\mathbf{q}$ and $-\mathbf{q}$ fields become random $^{3,6}$ and these terms vanish when averaged over time. Thus we obtain the structure function ${ }^{12}$ $\tau$ are reported by each curve. The solid curve corresponds to the Mie theory.
Author complimentary copy. Redistribution subject to AIP license or copyright, see http://apl.aip.org/apl/copyright.jsp

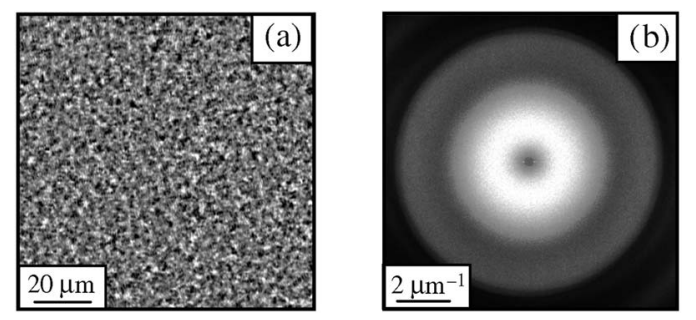

FIG. 2. (a) Differential heterodyne signal from $2.0 \mu \mathrm{m}$ diameter latex spheres diluted in density matched deuterated water at a lag time $\tau=3 \mathrm{~s}$. (b) Corresponding time-averaged 2D-structure function.

$$
\begin{aligned}
I(\mathbf{q}, \tau) & \equiv\left\langle|\delta F(\mathbf{q}, \tau)|^{2}\right\rangle_{t} \\
& =4 I(\mathbf{q})-4 \operatorname{Re}\left\{\left\langle E(\mathbf{q}, t) E^{*}(\mathbf{q}, t+\tau)\right\rangle_{t}\right\},
\end{aligned}
$$

which is given by the difference between the static intensity distribution $I(\mathbf{q})=\left\langle|E(\mathbf{q})|^{2}\right\rangle$ and the field-field correlation function $G_{E}(\mathbf{q}, \tau)=\operatorname{Re}\left\{\left\langle E(\mathbf{q}, t) E^{*}(\mathbf{q}, t+\tau)\right\rangle_{t}\right\}$. Clearly, $I(\mathbf{q}, \tau \rightarrow 0) \rightarrow 0$, while $I(\mathbf{q}, \tau \rightarrow \infty) \rightarrow I(\mathbf{q})$, with the crossover or correlation time $\tau_{c}$ depending on $|\mathbf{q}|$. Figure 2 shows a typical example of data obtained from a dilute solution of polystyrene spheres in density matched deuterated water. The particles were $2.0 \mu \mathrm{m}$ in diameter and the lag time was $\tau=3 \mathrm{~s}$. Because the sample is made of many particles, the differential heterodyne signal $\delta f(\mathbf{r}, t, \tau)$ appears to be uniformly speckled [Fig. 2(a)] and the corresponding 2D-structure function [Fig. 2(b)] exhibits an evident dip at $\mathbf{q}=(0,0)$, indicating the presence of higher and higher correlations as $q \rightarrow 0$. For isotropic samples, we can average $I(\mathbf{q}, \tau)$ over annular rings in the $q$ space and obtain a structure function $I(q, \tau)$ that depends only on $q=|\mathbf{q}|$. It is worth noticing that the averaging procedure, which involves a different number of pixels $N$ for each ring, corresponds to averaging $N$ independent $I(\mathbf{q}, \tau)$ functions. This occurs because the pixel size in the $q$ space $\left(q_{\min }=2 \pi / L\right)$ is equal to the $q$ size of the far-field speckles generated by an aperture equal to the size $L$ of the sensor. Thus, since $N \gg 1$, the technique is very robust from a statistical point of view.

The structure function $I(q, \tau)$ corresponding to the data of Fig. 2 is reported in Fig. 3. One can notice that $I(q, \tau)$ relaxes to its asymptotic behavior $(\tau \rightarrow \infty)$ with time scales that strongly decrease upon increasing the $q$ value. For

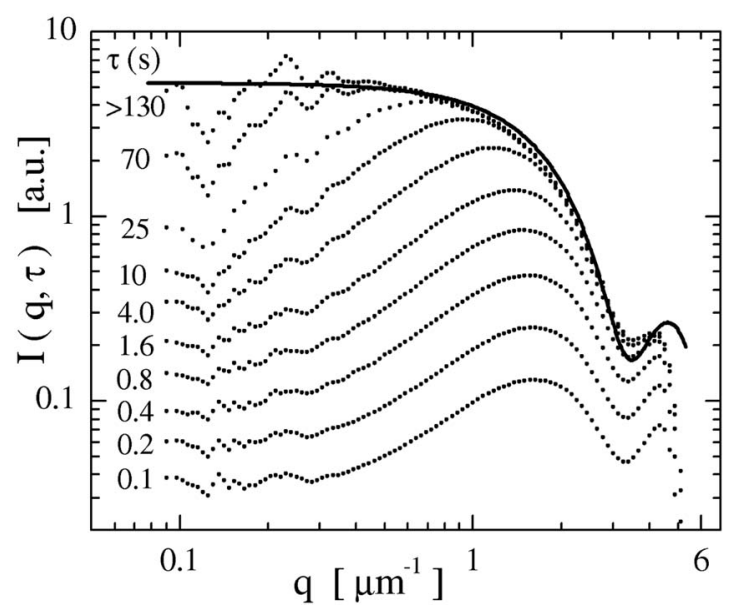

FIG. 3. Structure function obtained from a dilute solution of $2.0 \mu \mathrm{m}$ diameter polystyrene spheres in density matched deuterated water. The lag times $\tau$ are reported by each curve. The solid curve corresponds to the Mie theory. 


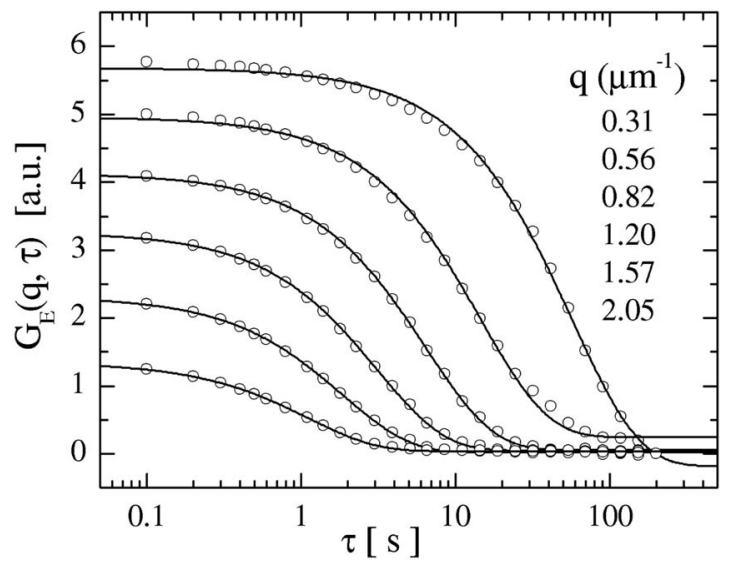

FIG. 4. Behavior of $G_{E}(q, \tau)$ vs $\tau$ for six different wavevectors (increasing from top to bottom). The sample was the same as that of Fig. 3. The solid curves are the best fittings of the data to Eq. (5).

$\tau \sim 100 \mathrm{~s}$ or larger, $I(q, \tau)$ approaches the static curve $I(q)$, which is consistent with the Mie theory (solid curve).

From Eq. (4), we recover $G_{E}(q, \tau)$ as the difference between $I(q)$ and $I(q, \tau)$ and fit it to the function

$$
G_{E}(q, \tau)=I(q) \exp \left[-D q^{2} \tau\right]+B(q),
$$

in which the amplitude $I(q)$, the particle translational diffusion coefficient $D$, and the baseline $B(q)$ can be used as floating parameters. When we analyze the data of Fig. 3 and fit the recovered $G_{E}(q, \tau)$ accordingly to Eq. (5), we obtain the results shown in Fig. 4, in which, for the sake of clarity, we reported only six curves. As evident, data (symbols) and fits (solid curves) match quite well to each others.

It should be pointed out that the largest range of $q$ 's actually usable with this technique is limited by the stability of the baseline $B(q)$, which might depend on $\tau$ as well. $B(q)$ is a background term, which accounts for both electronic noise (dark current, readout, shot noise, ect.) and optical/ mechanical noise (laser power fluctuations, mechanical/ thermal instabilities, air turbulence, presence of dust particles, etc.). While the first one is $\tau$ independent (and is usually negligible), the latter one tends to increase with $\tau$ and, for $\tau \geqslant 50-100 \mathrm{~s}$, may become comparable with $I(q)$. This occurs mainly at low $q$ 's, where the decay times are long, and at large $q \mathrm{~s}$, where $I(q)$ tends to zero. As a consequence, we restricted our analysis to a $q$ range inside which the $\tau$ dependence of the background term $B(q)$ could be neglected, corresponding, for our experimental conditions, to $0.3 \leqslant q \leqslant 3 \mu \mathrm{m}^{-1}$. Within this range, from the fitted values for $D$, by using the Stokes-Einstein relation, we recovered the particle diameters at the different wavevectors. This is shown in Fig. 5 for five diameters varying between 0.3 and $5.0 \mu \mathrm{m}$. The figure shows that, over most of the $q$ range $\left(\sim 0.5-2 \mu \mathrm{m}^{-1}\right)$, the diameters are recovered rather accurately $(\sim 5 \%)$ with deviations that become increasingly systematic at high $q$ 's and noisy at low q's. When compared to the (few) data taken with low-angle DLS and available in literature, ${ }^{2}$ our results appear to be quite comparable for what concerns both the $q$ range and the accuracy. However, our technique is simpler and definitely much more sensitive (see Ref. 5), allowing to study also submicron particles.

In conclusion, we have proposed a method for directly measuring the field-field correlation function $G_{E}(q, \tau)$ of the light scattered at low angle. With respect to the classical Author complimentary copy. Redistribution subject to AIP

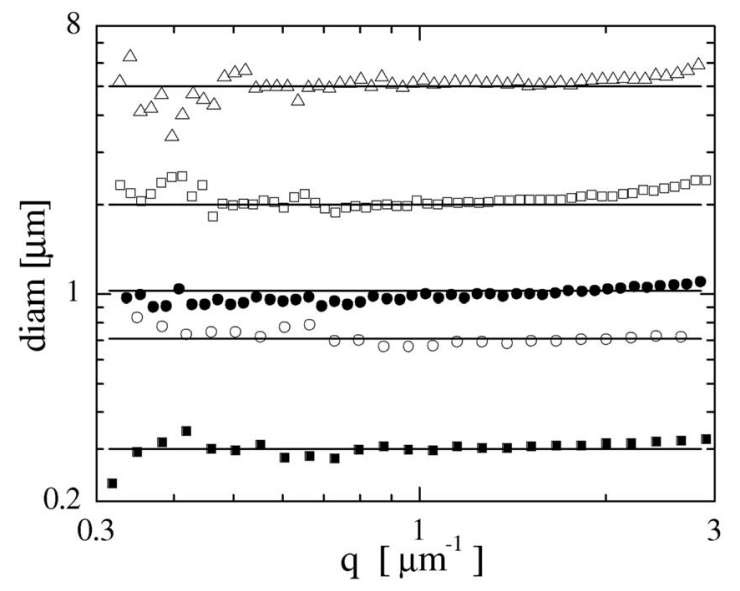

FIG. 5. Recovered diameters vs $q$ for various latex spheres diluted in density matched deuterated water. The certified particle diameters (indicated by the horizontal lines) were 5.0, 2.0, 1.02, 0.71, and $0.30 \mu \mathrm{m}$.

low-angle DLS, our method presents several advantages related to its heterodyne nature: (a) it adopts a much simpler optical layout, with no removal of the transmitted beam (b) it works without necessity of any blank measurement; (c) it is immune from stray-light problems that makes any system studied at low angles intrinsically nonergodic; (d) it measures directly $G_{E}(q, \tau)$ without passing via the Siegert relation, and therefore allowing the study of truly nonergodic systems. The main limitations of the technique are (a) the high optical/mechanical stability required for large lag times $(\tau \geqslant 50-100 \mathrm{~s})$ and (b) its intrinsic vulnerability against collective motions present in the sample (such as sedimentation or convective motions), which might obscure diffusion. However, if these motions are uniform, they can be measured as well, and, under some circumstances, the method here proposed can act also as a velocimetry technique. ${ }^{15,16}$ Works are in progress for ascertaining the possibility of simultaneous velocimetry and DLS measurements.

We thank Dario Pescini for early development of the technique and David S. Cannell for the critical reading of this manuscript.

${ }^{1}$ P. Y. Wong and P. Wiltzius, Rev. Sci. Instrum. 64, 2547 (1993).

${ }^{2}$ L. Cipelletti and D. Weitz, Rev. Sci. Instrum. 70, 3214 (1999).

${ }^{3}$ D. Brogioli, A. Vailati, and M. Giglio, Appl. Phys. Lett. 81, 4109 (2002).

${ }^{4}$ F. Ferri, D. Magatti, D. Pescini, M. A. C. Potenza, and M. Giglio, Phys. Rev. E 70, 041405 (2004).

${ }^{5}$ F. Scheffold and R. Cerbino, Curr. Opin. Colloid Interface Sci. 12, 50 (2007).

${ }^{6}$ M. Born and E. Wolf, Principles of Optics (Pergamon, New York, 1980), Chap. 12

${ }^{7}$ J. W. Goodman, Introduction to Fourier Optics, 2nd ed. (McGraw-Hill, New York, 1996), p. 87.

${ }^{8}$ M. Wu, G. Ahlers, and D. S. Cannell, Phys. Rev. Lett. 75, 1743 (1995).

${ }^{9}$ S. P. Trainoff and D. S. Cannell, Phys. Fluids 14, 1340 (2002).

${ }^{10}$ D. Brogioli, A. Vailati, and M. Giglio, Europhys. Lett. 63, 220 (2003).

${ }^{11}$ F. Croccolo, D. Brogioli, A. Vailati, M. Giglio, and D. S. Cannell, Appl. Opt. 45, 2166 (2006).

${ }^{12}$ F. Croccolo, D. Brogioli, A. Vailati, M. Giglio, and D. S. Cannell, Ann. N.Y. Acad. Sci. 1077, 365 (2006).

${ }^{13}$ R. Cerbino, L. Peverini, M. A. C. Potenza, A. Robert, P. Bosecke, and M. Giglio, Nat. Phys. 837, 238 (2008).

${ }^{14}$ J. W. Goodman, Statistical Optics (Wiley, New York, 1985), p. 79.

${ }^{15}$ M. D. Alaimo, D. Magatti, F. Ferri, and M. A. C. Potenza, Appl. Phys. Lett. 88, 191101 (2006).

${ }^{16}$ M. D. Alaimo, M. A. C. Potenza, D. Magatti, and F. Ferri, J. Appl. Phys. 102, 073113 (2007).

license or copyright, see http://apl.aip.org/apl/copyright.jsp 